MS32-P10

\section{A systematic structural study of halogenated 2-phenylbenzimidazoles}

Laura Nagyné Bereczki ${ }^{1}$, Zita Makó ${ }^{2}$, Dániel Fegyverneki ${ }^{1}$, Nóra

Veronika May ${ }^{1}$, Tibor Soós ${ }^{1}$, Petra Bombicz

1. Research Centre for Natural Sciences, Hungarian Academy of Sciences, Buadpest, Hungary

2. Faculty of Chemical Technology and Biotechnology, Budapest University of Technology and Economics, Budapest, Hungary

email: nagyne.bereczki.laura@ttk.mta.hu

The tunable interaction strength and the pronounced directionality make halogen bonding an eligible tool for crystal engineering. Due to the polarizability of the halogens, both nucleophilic and electrophilic regions can be found on the surface of covalently bound halogen atoms which allows them to form a variety of secondary interactions in the solid state $^{1}$. The interaction strength scales with the polarizability of the halogen atom in a relatively wide range.

The main objective of this study is to determine how and to what extent the type and the position of the halogen substituent may affect the crystal structure if the other influencing factors are reduced as far as possible.

The crystal structures of halogenated 2-phenlybenzimidazoles are compared. The 2-phenylbenzimidazoles are substituted on the phenyl ring in ortho, meta and para positions or simultaneously in two different positions by fluorine, chlorine and bromine. In the studied crystal structures, the halogens form R-X...X-R' and R-X...Pi type halogen bonds and R-H...X-R' hydrogen bonds.

8 structures from the CSD (JEYTUD, MINHOI, TUDXIB, LUJWIW01, DETKIX, GOLNOM, QERTIR, SEZNUH) and 3 new halogenated 2-phenylbenzimidazole structures are compared and analyzed. Correlations between the halogen atom positions, secondary interactions and crystallographic parameters are analyzed by statistical methods.

2-Phenylbenzimidazole has a rigid structure that can be twisted around the benzimidazole-phenyl axis. The solid state interactions of halogenated 2-phenylbenzimidazoles are relatively simple which makes them good model compounds for this type of study. Their structure is basically determined by only one intermolecular interaction that is significantly stronger than every other secondary interaction in the crystal lattice. Namely, N-H...N hydrogen bonded chains organize the crystal structures and only a few different arrangements of these chains are possible. However, these structures are flexible to some extent, and permit the alteration of the lattice parameters.

Most of the studied halogenated 2-phenylbenzimidazoles are isostructural. The exchange of the different halogen substituents causes slight changes in the crystal packing that on the whole results in a significant change in the lattice parameters. In the case of the different halogen substituents, the angle between the neighboring benzimidazole moieties varies from $60^{\circ}$ to $130^{\circ}$. The length of the unit cell axes correlates with this angle and a nearly twofold increase can be observed in the length of the $c$ axis in the series of isostructural crystals.
Financial support of the National Research, Development and Innovation Office (NKFIH) through OTKA K124544 is gratefully acknowledged.

References:

[1] Cavallo, G. et al. (2016). Chem. Rev. 116. 2478-2601.

Keywords: supramolecular interaction, crystal engineering, benzimidazole 